Z Gerontol Geriat 2022 · 55:590-596 https://doi.org/10.1007/s00391-021-01959-8

Eingegangen: 1. Dezember 2020

Angenommen: 9. Juli 2021

Online publiziert: 19. August 2021

(c) Springer Medizin Verlag GmbH, ein Teil von Springer Nature 2021

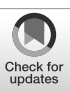

\section{Kommen die alten Ängste wieder?}

\author{
Eine Untersuchung zum Erleben der Corona-Krise bei \\ Überlebenden des Hamburger Feuersturms (1943)
}

Ulrich Lamparter ${ }^{1} \cdot$ Hendrik Althoff ${ }^{2} \cdot$ Christa Holstein ${ }^{1} \cdot$ Sabine Lucassen ${ }^{1}$. Ursula Pilz' • Ute Rippel-Lau'

${ }^{1}$ Adolf-Ernst-Meyer-Institut für Psychotherapie, Hamburg, Deutschland

${ }^{2}$ Fachbereich Geschichte, Universität Hamburg, Hamburg, Deutschland

Hintergrund: Im Rahmen des „Erinnerungswerk Hamburger Feuersturm 1943“ wurde bis März 2020 ein Fundus von 120 lebensgeschichtlichen Interviews mit Überlebenden der Luftangriffe auf Hamburg im Juli 1943 angelegt.

Ziel der Arbeit: Unter dem Eindruck des ersten Lockdowns im Rahmen der Coronakrise im Frühjahr 2020 stellte sich die Frage, ob die Kriegserfahrungen der Zeitzeugen im Hamburger Feuersturm den Umgang mit der Krise prägen.

Methodik: Diese Zeitzeugen wurden im Mai und im Dezember 2020 in einem Fragebogen zu ihrem Erleben der Coronakrise befragt. Weiter liegen Befunde aus Telefongesprächen mit Mitgliedern einer kontinuierlichen Gesprächsgruppe vor. Ergebnisse: Im Mai 2020 und im Dezember 2020 antworteten 98 (82\%) und 77 (64\%) der Angeschriebenen, 58 (45) Frauen und 40 (32) Männer mit einem Durchschnittsalter von $86,5(86,7)$ Jahren. Sie stellen sich zu beiden Zeitpunkten überwiegend als relativ stabil und zuversichtlich dar. Im Vordergrund des Coronaerlebens steht die Kontakteinschränkung, weniger die Sorge um die eigene Gesundheit. Stark werden negative wirtschaftliche Folgen für Deutschland befürchtet. Auf der höchsten Stufe der Zustimmung bejahen $13 \%$ der Befragten, dass die gegenwärtige Krise sie an die Erfahrungen im Hamburger Feuersturm erinnert. Telefongespräche verdeutlichen, wie die Erfahrung der Kriegs- und Nachkriegszeit eine Leiterfahrung für das Erleben und den Umgang mit der Coronakrise darstellen kann.

Diskussion: Die Befunde weisen auf generationstypische, mit den Kriegserfahrungen des Zweiten Weltkriegs verbundene Erfahrungsmuster in der älteren Bevölkerung hin, die das Erleben der Coronakrise prägen.

\title{
Schlüsselwörter
}

Coronakrise · Luftkrieg · Psychische Folgen des Zweiten Weltkriegs · Bewältigung

\section{Einleitung}

Die Coronapandemie und der damit verbundene erste Lockdown im Frühjahr 2020 mit seinen massiven Kontakteinschränkungen haben viele Menschen unvorbereitet getroffen. Für die Altenheime und Pflegeeinrichtungen galten besonders strenge Regeln der Kontaktvermeidung, da die Gruppe der Alten als Hochrisikogruppe galt. Bei dieser Altersgruppe handelt es sich um die Generation der "Kriegskinder", also der Menschen, von denen viele in Deutschland als Kinder den Zweiten Weltkrieg erlebt haben. Es stellt sich die Frage, wie sie diese die Coronakrise erleben und mit ihr umgehen, und ob sie die Kriegszeit damals mit der jetzigen Krisenzeit in Verbindung bringen: Kehren in der Isolation der Coronatage alte Ängste aus den „Bombennächten" wieder? Um diese Fragen differenziert beantworten zu können, erscheinen Untersuchungen an zeitgeschichtlich definierten Gruppen, wie 
etwa den Überlebenden des „Hamburger Feuersturms" sinnvoll. Die als „Operation Gomorrha" bezeichneten Luftangriffe der Alliierten im Juli 1943 zerstörten weite Teile der Stadt [7] und haben sich als „Hamburger Feuersturm" im kollektiven Gedächtnis verankert [10].

Ihrem Erleben und der Frage nach der transgenerationalen Weitergabe war das interdisziplinäre Forschungsprojekt "Zeitzeugen des Hamburger Feuersturms und ihre Familien" [4] gewidmet, das im Zuge der "Kriegskind-Forschung" [9] entstand und 60 Zeitzeugen und ihre Familien untersucht hat. Dort zeigte sich bei vielen Zeitzeugen neben typischen posttraumatischen Symptomen (Angst vor Bränden, Albträume, reizassoziierte Überempfindlichkeiten und Ängste) überwiegend eine fortbestehende Grunderschütterung im Sinne einer hintergründigen psychischen Labilität trotz äußerlicher Stabilität durch die Erfahrung im Feuersturm [2]. Ihre Bewältigung wurde stark durch die Erfahrungen der Nachkriegszeit beeinflusst [5].

Das „Erinnerungswerk Hamburger Feuersturm“ (EHF 1943) [www.ehf-1943.de] sammelt als praktische Konsequenz aus diesem Projekt weiter fortlaufend Interviews mit den heute hochbetagten Überlebenden. Mittlerweile wurden im Erinnerungswerk 120 lebensgeschichtliche Interviews von psychodynamisch orientierten Psychotherapeuten durchgeführt. Es handelt sich um die Geburtsjahrgänge 1923-1942; die älteste Interviewte war zum Zeitpunkt des Gesprächs 96 Jahre alt. 14 von innen lebten im Heim oder in einer Pflegeeinrichtung. Weiter wird im Erinnerungswerk eine kontinuierliche Gesprächsgruppe für Zeitzeuginnen und Zeitzeugen des Hamburger Feuersturms angeboten.

Als die Coronakrise im Frühjahr 2020 diese Arbeit unterbrach, haben wir uns gefragt, wie „unsere Zeitzeugen" mit der Situation des Lockdowns umgehen, und inwieweit es hier zu einem bedrängenden und möglicherweise destabilisierenden Wiedererinnern der existenziellen Grenzerfahrung im Hamburger Feuersturm gekommen war. Erneut wurden diese Fragen im Lockdown im Dezember 2020 vor den bevorstehenden Weihnachtstagen aufgeworfen. Es stellten sich insbesondere folgende Fragen:
- Wie geht es den Zeitzeugen gegenwärtig unter den Bedingungen des Lockdowns?

- Was macht ihnen besonders zu schaffen?

- Wie schätzen Sie die allgemeine Lage ein?

- Werden die Zeitzeugen durch Coronakrise und Lockdown an ihre Erfahrungen in der Kriegs- und Nachkriegszeit erinnert?

\section{Methodik}

\section{Telefongespräche}

Eine kontinuierliche Gesprächsgruppe mit Zeitzeugen im Rahmen des Erinnerungswerk Hamburger Feuersturm bestand im März 2020 aus 9 Mitgliedern (4 Frauen, 5 Männer). Die Infektionszahlen waren noch relativ gering, es bestand jedoch ein Mangel an Masken und Infektionsmitteln, und Kenntnisse über das Virus waren wenig verbreitet. Am 24.03.2020 führte die Leiterin der Gruppe (S. L.) 7 Telefongespräche, um die Unterbrechung der Gruppe wegen des "Corona-Lockdowns" anzukündigen und sich nach dem Ergehen der Gruppenmitglieder zu erkundigen. Nach den Telefonaten wurden kurze Gesprächsnotizen angefertigt.

Zahlreiche Äußerungen der Gruppenteilnehmer zeigen die Art der Bezugnahme desErlebens der Coronakrisezu den Kriegsund Nachkriegserfahrungen und illustrieren die später in Fragebogen erhobenen Befunde.

\section{Fragebogenuntersuchung im Mai} 2020

Im Mai 2020 wurde an alle 120 Zeitzeugen, die bisher im Rahmen des Erinnerungswerks interviewt worden waren, ein Fragebogen verschickt. Zu diesem Zeitpunkt war die erste Welle des Infektionsgeschehens schon deutlich im Abklingen. Es war freilich noch ungewiss, wie „es weitergehen würde". Der Lockdown hatte schon über 6 Wochen angedauert.

Der Fragebogen bestand aus 23 Fragen zu den Themen des persönlichen Umgangs mit der Krise und dabei erlebten Belastungen, dem Umgang mit der Krise vor dem Hintergrund eigener Erfahrungen in
Krieg und Nachkriegszeit sowie zum gesellschaftlichen Umgang mit der Krise, zu Zukunftssorgen und zu erlebten Stützen. Es wurden 5 Antwortkategorien vorgegeben (sehr schlecht $(=1)$, schlecht (=2), mittelmäßig $(=3)$, gut $(=4)$, sehr gut $(=5)$; bzw. nein $(=1)$, eher nein $(=2)$, teils-teils $(=3)$, eher ja (=4), ja (=5)). Zu zwei Fragen wurden Freitextantworten erbeten.

Es antworteten 98 (81,7\%), 58 Frauen und 40 Männer, darunter 3 Heimbewohner, mit einem mittleren Alter von 86,5 Jahren, $S D= \pm 3,72$ ). Sie entstammten überwiegend den Geburtsjahrgängen 1932-1938 und waren während des Feuersturms 1943 im Mittel 8 Jahre alt gewesen. Insgesamt 5 Bogen (4\%) kamen als unzustellbar zurück, weil die Adressaten mittlerweile verstorben waren.

Dabei erhielten wir von 93 Zeitzeugen (oder $78 \%$ ) schon im Zeitraum vom 15. Mai bis zum 20. Juni eine Rückmeldung. Ende Juni sanken die Infektionszahlen deutlich, und es traten erste Grenzöffnungen und Lockerungen in Kraft. Alte Menschen galten freilich weiter als besonders gefährdet.

\section{Fragebogenuntersuchung im Dezember 2020}

Mitte Dezember 2020 wurde unter der zweiten Welle der Pandemie ein weiterer Lockdown verordnet. Es standen nun zwar Masken zur Verfügung, und es waren die ersten Impfstoffe entwickelt worden, aber die Infektionszahlen waren massiv angestiegen. Erneut haben wir uns mittels des entwickelten Fragebogens nach dem Ergehen der Zeitzeuginnen und Zeitzeugen zu erkundigt und dabei einige aktuelle Zusatzfragen hinzugefügt. Auf diese Umfrage antworteten jetzt 77 (64\%) der Angeschriebenen, 45 Frauen und 32 Männer mit einem Durchschnittsalter von 86,7 Jahren.

\section{Ergebnisse}

\section{Befunde aus den Telefongesprächen}

Allen Zeitzeuginnen und Zeitzeugen schien es generell wichtig, die „Fassung zu bewahren“. In 5 Telefonaten wurde die Kriegszeit thematisiert. Die Gesprächspartner kamen im Umgang mit dem Lockdown auf Haltungen und Erfahrun- 


\section{Originalien}

\begin{tabular}{|c|c|c|c|c|c|c|c|c|c|}
\hline \multicolumn{2}{|c|}{ Umfragen zur Coronapandemie } & \multicolumn{2}{|c|}{\begin{tabular}{l|l|} 
Mittel- & Standard- \\
wert & abwei- \\
$(\mathrm{m})$ & chung (s) \\
\end{tabular}} & $\begin{array}{l}\text { Korrelation } \\
\text { (r) mit Item } \\
\text { Nr. } 11\end{array}$ & \multirow[t]{2}{*}{$p$} & \multirow{2}{*}{$\begin{array}{l}\text { Mittel- } \\
\text { wert } \\
\text { (m) } \\
\text { Umfrag }\end{array}$} & $\begin{array}{l}\text { Standard- } \\
\text { abwei- } \\
\text { chung (s) }\end{array}$ & \begin{tabular}{l|} 
Korrelation \\
(r) mit Item \\
Nr. 11 \\
\end{tabular} & \multirow[t]{2}{*}{$p$} \\
\hline Nr. & Fragetext & \multicolumn{3}{|c|}{ Umfrage $1(n=98)$ Mai 2020} & & & \multicolumn{2}{|c|}{ Umfrage $2(n=77)$ Dezember 2020} & \\
\hline 1 & $\begin{array}{l}\text { Wie geht es Ihnen gegenwärtig gesund- } \\
\text { heitlich? }\end{array}$ & 3,57 & 0,82 & 0,08 & 0,41 & 3,51 & 0,66 & 0,05 & 0,60 \\
\hline 2 & $\begin{array}{l}\text { Haben Sie persönlich Angst, sich am Coro- } \\
\text { navirus anzustecken? }\end{array}$ & 2,17 & 0,93 & 0,10 & 0,35 & 2,46 & 0,98 & 0,33 & $<0,001$ \\
\hline 3 & $\begin{array}{l}\text { Haben Sie für sich selbst vorgesorgt und } \\
\text { Vorräte angelegt? }\end{array}$ & 2,15 & 1,30 & 0,13 & 0,22 & 2,15 & 1,31 & 0,08 & 0,41 \\
\hline 4 & $\begin{array}{l}\text { Halten Sie sich streng an die "Coronare- } \\
\text { geln“ (Abstandsgebote, Kontaktsperre, } \\
\text { Hygieneregeln usw.)? }\end{array}$ & 4,41 & 0,76 & 0,11 & 0,28 & 4,47 & 0,75 & 0,16 & 0,11 \\
\hline 5 & Mussten Sie Ihre Kontakte einschränken? & 4,07 & 1,16 & 0,09 & 0,40 & 4,01 & 1,13 & 0,07 & 0,53 \\
\hline 6 & Ist Ihnen das schwergefallen? & 3,54 & 1,45 & 0,13 & 0,21 & 3,89 & 1,26 & 0,12 & 0,23 \\
\hline 7 & Falls ja, was war hier das Schwerste? & \multicolumn{8}{|c|}{ Freitextantworten } \\
\hline 8 & $\begin{array}{l}\text { Haben Sie körperliche oder seelische Sym- } \\
\text { ptome, die Sie auf diese Krise zurückfüh- } \\
\text { ren? }\end{array}$ & 1,84 & 1,17 & 0,12 & 0,25 & 1,92 & 1,05 & 0,10 & 0,32 \\
\hline 9 & $\begin{array}{l}\text { Hat Ihr Schlaf unter der derzeitigen Krise } \\
\text { gelitten? }\end{array}$ & 1,69 & 1,10 & 0,26 & 0,01 & 1,90 & 1,07 & 0,32 & 0,001 \\
\hline 10 & $\begin{array}{l}\text { Haben Sie angesichts der gegenwärti- } \\
\text { gen Bedrohung durch Corona Ängste, } \\
\text { die schlimmer sind, als sie eigentlich sein } \\
\text { müssten, und gegen die Sie sich nicht weh- } \\
\text { ren können? }\end{array}$ & 1,61 & 1,02 & 0,27 & 0,01 & 1,74 & 0,98 & 0,40 & $<0,001$ \\
\hline 11 & $\begin{array}{l}\text { Erinnert die gegenwärtige Krise Sie an Ihre } \\
\text { Erfahrungen im Hamburger Feuersturm? }\end{array}$ & 2,35 & 1,39 & - & - & 2,48 & 1,49 & - & - \\
\hline 12 & $\begin{array}{l}\text { Erinnert die gegenwärtige Krise Sie an Ihre } \\
\text { Erfahrungen in der Nachkriegszeit? }\end{array}$ & 2,65 & 1,50 & 0,58 & $<0,001$ & 2,64 & 1,44 & 0,64 & $<0,001$ \\
\hline 13 & $\begin{array}{l}\text { Empfinden Sie die Coronakrise im Ver- } \\
\text { gleich zur Kriegs- und Nachkriegszeit als } \\
\text { eher harmlos? }\end{array}$ & 2,93 & 1,48 & $-0,30$ & 0,01 & 2,57 & 1,39 & $-0,10$ & 0,16 \\
\hline 14 & $\begin{array}{l}\text { Haben Sie den Eindruck, die Coronakrise } \\
\text { wird von der Politik dramatisiert? }\end{array}$ & 1,96 & 1,06 & 0,05 & 0,66 & 1,91 & 1,16 & $-0,10$ & 0,18 \\
\hline 15 & $\begin{array}{l}\text { Haben Sie den Eindruck, dass die Politik } \\
\text { sich ausreichend um die älteren Menschen } \\
\text { kümmert? }\end{array}$ & 3,21 & 1,12 & $-0,10$ & 0,30 & 3,33 & 1,13 & $-0,10$ & 0,56 \\
\hline 16 & $\begin{array}{l}\text { Haben Sie den Eindruck, dass die jünge- } \\
\text { ren Menschen gut verstehen, wie es den } \\
\text { Älteren in der Krise geht? }\end{array}$ & 2,76 & 1,2 & $-0,1$ & 0,15 & 2,82 & 1,27 & $-0,10$ & 0,22 \\
\hline 17 & $\begin{array}{l}\text { Fürchten Sie ernsthafte volkswirtschaftli- } \\
\text { che Schäden für Deutschland? }\end{array}$ & 4,14 & 0,96 & 0 & 0,98 & 4,25 & 0,9 & 0 & 0,87 \\
\hline 18 & Belasten Sie solche Befürchtungen? & 3,08 & 1,22 & 0,07 & 0,50 & 3,11 & 1,24 & 0,20 & 0,05 \\
\hline 19 & $\begin{array}{l}\text { Fürchten Sie ernste wirtschaftliche Fol- } \\
\text { gen auch für sich persönlich (z. B. Inflation, } \\
\text { Besteuerung des Ersparten, Rentenkürzun- } \\
\text { gen)? }\end{array}$ & 2,86 & 1,31 & 0,09 & 0,36 & 3,01 & 1,38 & 0 & 0,88 \\
\hline 20 & Belasten Sie solche Befürchtungen? & 2,43 & 1,26 & 0,14 & 0,18 & 2,57 & 1,35 & 0,12 & 0,25 \\
\hline 21 & $\begin{array}{l}\text { Haben Sie die Erwartung, dass Deutsch- } \\
\text { land gut durch die Krise kommen wird? }\end{array}$ & 3,94 & 0,91 & 0 & 1,00 & 3,71 & 0,84 & 0 & 0,94 \\
\hline 22 & $\begin{array}{l}\text { Gibt es etwas, das Ihnen jetzt in der Krise } \\
\text { besonders hilft? }\end{array}$ & \multicolumn{8}{|c|}{ Freitextantworten } \\
\hline 23 & $\begin{array}{l}\text { Sind Ihnen hier Ihre Erfahrungen in der } \\
\text { Kriegs- oder Nachkriegszeit nützlich? }\end{array}$ & 3,63 & 1,53 & 0,26 & 0,01 & 3,61 & 1,45 & 0,24 & 0,02 \\
\hline 24 & $\begin{array}{l}\text { Macht Ihnen die Dauer der Krise zu schaf- } \\
\text { fen? }\end{array}$ & - & - & - & - & 3,75 & 1,03 & 0,02 & 0,84 \\
\hline
\end{tabular}




\begin{tabular}{|c|c|c|c|c|c|c|c|c|c|}
\hline \multicolumn{2}{|c|}{ Umfragen zur Coronapandemie } & $\begin{array}{l}\text { Mittel- } \\
\text { wert } \\
\text { (m) }\end{array}$ & $\begin{array}{l}\text { Standard- } \\
\text { abwei- } \\
\text { chung (s) }\end{array}$ & $\begin{array}{l}\text { Korrelation } \\
\text { (r) mit Item } \\
\text { Nr. } 11\end{array}$ & $p$ & $\begin{array}{l}\text { Mittel- } \\
\text { wert } \\
\text { (m) }\end{array}$ & $\begin{array}{l}\text { Standard- } \\
\text { abwei- } \\
\text { chung (s) }\end{array}$ & $\begin{array}{l}\text { Korrelation } \\
\text { (r) mit Item } \\
\text { Nr. } 11\end{array}$ & $p$ \\
\hline Nr. & Fragetext & \multicolumn{4}{|c|}{ Umfrage $1(n=98)$ Mai 2020} & \multicolumn{4}{|c|}{ Umfrage $2(n=77)$ Dezember 2020} \\
\hline 25 & $\begin{array}{l}\text { Haben Sie unter Einsamkeit oder Alleinsein } \\
\text { zu leiden? }\end{array}$ & - & - & - & - & 2,33 & 1,31 & 0,02 & 0,86 \\
\hline 26 & $\begin{array}{l}\text { Mussten Sie selbst in Quarantäne oder } \\
\text { waren besonderer Kontaktbeschränkung } \\
\text { ausgesetzt? }\end{array}$ & - & - & - & - & 1,03 & 0,15 & $-0,10$ & 0,21 \\
\hline 27 & $\begin{array}{l}\text { Ist jemand aus Ihrem persönlichen Umfeld } \\
\text { am Coronavirus erkrankt? }\end{array}$ & - & - & - & - & 1,14 & 0,35 & 0,23 & 0,02 \\
\hline 28 & $\begin{array}{l}\text { Ist jemand aus Ihrem persönlichen Umfeld } \\
\text { am oder mit dem Coronavirus verstorben? }\end{array}$ & - & - & - & - & 1,04 & 0,19 & 0,19 & 0,06 \\
\hline 29 & $\begin{array}{l}\text { Werden Sie selbst sich impfen lassen, so- } \\
\text { bald ein Impfstoff zur Verfügung steht? }\end{array}$ & - & - & - & - & 4,03 & 1,30 & $-0,10$ & 0,45 \\
\hline 30 & $\begin{array}{l}\text { Fühlen Sie sich gegenwärtig durch die } \\
\text { Coronapandemie stärker belastet als im } \\
\text { Frühjahr während des ersten Lockdowns? }\end{array}$ & - & - & - & - & 3,23 & 1,40 & 0,15 & 0,14 \\
\hline 31 & $\begin{array}{l}\text { Sind die coronabedingten Einschränkun- } \\
\text { gen zu Weihnachten für Sie besonders } \\
\text { schwer auszuhalten? }\end{array}$ & - & - & - & - & 2,59 & 1,37 & 0,16 & 0,11 \\
\hline 32 & $\begin{array}{l}\text { Ist für Sie das Feuerwerk zu Silvester mit } \\
\text { Erinnerungen an den Feuersturm verbun- } \\
\text { den? }\end{array}$ & - & - & - & - & 2,33 & 1,73 & 0,18 & 0,09 \\
\hline
\end{tabular}

gen in der Kriegs- und Nachkriegszeit zurück und schienen diese als Folie der Bewältigung, Einordung und Relativierung der Krise zu nutzen. Gelegentlich schienen projektive Elemente bzw. Vorurteile die Bewältigung zu stabilisieren. Im Eindruck der Gruppenleiterin wurden auch untergründige Beunruhigungen und drohende Instabilitäten spürbar, aber auch ein Stolz, dass man schon Schlimmeres erlebt und überstanden hat.

So fühlt sich eine Zeitzeugin (86 Jahre) an die Ausgangssperre 1945 erinnert, „als die Engländer kamen und die Lebensmittelregale leer waren“. Eine andere Zeitzeugin (80 Jahre) verweist auf die in der Kriegs- und Nachkriegszeit eingeprägten Muster und das vorausschauende Anlegen von Vorräten: "Manches muss man jetzt eben hinten anstellen“. Das sei für sie aber kein Problem. Das kenne sie ja noch. Vorräte habe sie immer ausreichend im Keller: „Das steckt in uns, für Krisenzeiten immer Vorsorge zu treffen“. Ein Zeitzeuge (81 Jahre) betont, wie wichtig es ist, auch "Glück" zu haben, eine Einstellung, der wir in unseren Untersuchungen zur Rückschau auf das Überleben im Feuersturm häufig begegnet sind [3]: „Wenn man in Trümmern liegt, braucht man auch Glück, man kann sich anpassen und im- provisieren, aber Glück braucht man auch." Er betont auch die Fähigkeit, Mängel auszuhalten. „Es ist ja auch kein Drama, wenn es mal an etwas fehlt." Vielleicht lerne die nachfolgende Generation jetzt auch, dass man nicht in Panik geraten müsse, „wenn man mal kein Brot im Haus hat".

Für einen anderen Zeitzeugen (79 Jahre) ist dagegen der Hunger zentral. Er habe sich immer gesagt: bloß nie wieder hungern. Er ist froh, jetzt nicht hungern zu müssen. Er bezieht sich in seinem Erleben des Lockdowns nicht nur auf den Nahrungsmangel der Nachkriegszeit, sondern auf die „gesamte Kriegszeit" seit 1943, „da durften wir auch nicht raus".

Immer wieder werden in den Telefonaten die „Jungen“ erwähnt, die so etwas ja nicht gewohnt seien: „Für die Jungen ist es ja das erste Mal, dass sie zum Nachdenken kommen", meint eine Zeitzeugin (92 Jahre). Beim Einkaufen in der Kassenschlange habe sie kürzlich eine junge Frau zu ihrer Freundin sagen gehört: „Ich bin so wütend und verzweifelt. Ich hatte eine Theaterkarte, und jetzt fällt das aus". Da habe sie sich ins Gespräch gemischt: „Nun machen Sie mal nicht so ein Gesicht! Wir haben damals gehungert und die Bomben auf den Kopf gekriegt. Sie haben doch nicht ernsthaft was auszustehen, nur weil die Theater- vorstellung ausfällt, mein Kind“. Für einen anderen Zeitzeuge (82 Jahre), der seine Aktivität in Hamburger Schulen durchaus bewusst als eine Art Selbsttherapie begreift, ist es v.a. schwer zu ertragen, dass dies nun unter Coronabedingungen ins Stocken geraten ist.

\section{Analyse der Fragebogen}

- Tab. 1 zeigt die Ergebnisse des Fragebogenrücklaufs zu beiden Zeitpunkten im Mai und Dezember 2020. Die Frage 11: „Erinnert die gegenwärtige Krise Sie an Ihre Erfahrungen im Hamburger Feuersturm?" thematisiert die Erfahrungen im Feuersturm. Zu diesem Item wurde die Korrelation mit den anderen Items des Fragebogens berechnet.

Zum allgemeinen Umgang mit der Coronakrise geben die Befragten in der ersten Umfrage im Mai 2020 an, dass es ihnen gesundheitlich relativ gut geht $(m=3,57)$. Sie berichten, die "Coronaregeln“ $(m=4,40)$ genau zu befolgen. Die damit verbundenen Kontakteinschränkungen ( $m=4,07)$ auf sich zu nehmen, ist überwiegend schwergefallen $(m=3,54)$. Weniger geben die Befragen an, Vorräte angelegt zu haben $(m=2,17)$. Obwohl sie der "Risikogruppe" angehören, ist die 
Tab. 2 Freitextangaben zu Frage 7 (65 Fragebogen; 66,3\% der Rücksendungen) (Mai 2020) (Mehrfachantworten möglich)

\begin{tabular}{|l|l|}
\hline Unterbrochene Kontakte & $n$ \\
\hline $\begin{array}{l}\text { Familienangehörige, Freunde } \\
\text { und Bekannte nicht treffen zu } \\
\text { können }\end{array}$ & 24 \\
\hline $\begin{array}{l}\text { Verlust von gewohnten Aktivi- } \\
\text { täten oder Reisen }\end{array}$ & 14 \\
\hline $\begin{array}{l}\text { Nicht-umarmen-Können in der } \\
\text { Familie }\end{array}$ & 10 \\
\hline $\begin{array}{l}\text { Allgemeine Kontakt- und Be- } \\
\text { suchsreduktion }\end{array}$ & 8 \\
\hline $\begin{array}{l}\text { Wichtige Geburtstage nicht in } \\
\text { der Familie feiern zu können }\end{array}$ & 4 \\
\hline Erleben von Einsamkeit und Restriktion \\
\hline Alleinsein & 4 \\
\hline $\begin{array}{l}\text { Eigene Krankenbesuche sind } \\
\text { nicht möglich }\end{array}$ & 4 \\
\hline Erleben von Eingesperrtsein & 1 \\
\hline Kein Ausgang & 1 \\
\hline Kontakt nur am Telefon & 1 \\
\hline Zu wenig Bewegung & 1 \\
\hline Explizit „nicht viel vermisst" & 3 \\
\hline
\end{tabular}

Angst vor eigener Ansteckung ebenso relativ gering ausgeprägt ( $m=2,17)$. Körperliche oder seelische Symptome werden in noch geringerem Ausmaß auf die Coronakrise zurückgeführt $(m=1,84)$, zu Schlafstörungen kommt es kaum $(m=1,69)$, und über vermehrte Ängste wird vergleichsweise wenig berichtet $(m=1,61)$. Insgesamt stellen sich die Befragten also stabil und wenig beeinträchtigt dar.

Die Durchschnittswerte der Angaben zum historischen Vergleich liegen im mittleren Bereich. Dies gilt sowohl für die Wiedererinnerung an die Nachkriegszeit $(m=2,65)$ als auch an den Feuersturm $(\mathrm{m}=2,35)$.

Bei den Angaben zum gesellschaftlichen Umgang mit der Krise und zu Zukunftserwartungen stehen wirtschaftliche Sorgen im Vordergrund: Überwiegend wird befürchtet, dass es zu volkswirtschaftlichen Schäden für Deutschland kommt $(m=4,13)$. Diese belasten $(m=3,08)$, dabei ist die Sorge um die eigene wirtschaftliche Zukunft weniger ausgeprägt $(m=2,86)$. Überwiegend wird erwartet, dass Deutschland gut durch die Krise kommen wird $(m=3,94)$.

Die eigenen Erfahrungen in Kriegsund Nachkriegszeit werden im Umgang
Tab. 3 Freitextangaben zur Frage 22 in 88 Fragebogen; (89,8\% der Rücksendungen) (Mai 2020) (Mehrfachantworten möglich)

\begin{tabular}{|l|l|}
\hline $\begin{array}{l}\text { Stützendes Erleben in Partner- } \\
\text { schaft und Familie }\end{array}$ & $n$ \\
\hline Familie allgemein & 8 \\
\hline Kinder & 8 \\
\hline Partnerschaft & 4 \\
\hline Hund & 2 \\
\hline Enkel & 1 \\
\hline
\end{tabular}

Eigene Einstellungen

\begin{tabular}{|l|l}
\hline Optimismus, Glaube und Zuver- & 10
\end{tabular} sicht

$\begin{array}{ll}\text { Kraft aus den eigenen Erfahrun- } & 7\end{array}$ gen in Krieg und Nachkriegszeit

\begin{tabular}{|l|l} 
Erfahrung und Wissen & 5 \\
\hline
\end{tabular}

Eigenes Gesundheitsverhalten und Vorsicht

Ruhe bewahren und nicht zu viel nachdenken

Sozialer Zusammenhalt und günstige eigene Aktivitäten und Lebensumstände

\begin{tabular}{|l|l|}
\hline $\begin{array}{l}\text { Nachbarn, Hilfe im Alltag, Mög- } \\
\text { lichkeit, selbst zu helfen }\end{array}$ & 12 \\
\hline Natur und Bewegung, Hobby & 10 \\
\hline $\begin{array}{l}\text { Kommunikation über Telefon, } \\
\text { E-Mail oder WhatsApp }\end{array}$ & 8 \\
\hline Freundschaften & 5 \\
\hline Gute eigene Lebenssituation & 5 \\
\hline Vertrauen in die Politik & 4 \\
\hline $\begin{array}{l}\text { Hoffnung auf Besserung und } \\
\text { Lockerung der Maßnahmen }\end{array}$ & 3 \\
\hline $\begin{array}{l}\text { Sinnlicher Genuss im Rahmen des } \\
\text { Möglichen }\end{array}$ & 2 \\
\hline Gottesdienstbesuche & 1 \\
\hline Gespräche & 1 \\
\hline Ruhe und Schlaf & 1 \\
\hline Keine besondere Hilfe & 4 \\
\hline
\end{tabular}

mit der Krise als nützlich erlebt $(m=3,62)$. Dieser Wert liegt deutlich höher als die Durchschnittswerte der Items 11 und 12, die eine belastende und ängstigende Wiedererinnerung thematisieren.

\section{Analyse der Textantworten in der} ersten Befragung (Mai 2020)

\section{Erleben der Kontakteinschränkung}

Auf die Frage 7 (Was war das Schwerste bei der Einhaltung der Kontakteinschränkungen?) wurde in 65 Fragebogen geantwortet. Die Inhalte der Antworten ließen sich thematisch wie folgt gruppieren (• Tab. 2).
Die Antworten betonen die Personen, mit denen Kontakte nicht möglich oder unterbunden waren. Besonders schmerzlich wurde erlebt, "dass man sich in der Familie nicht mehr umarmen kann", ebenso wenn eigene Besuche im Pflegeheim nicht möglich waren.

\section{Hilfe in der Krise}

Zu der Frage 22: Gibt es etwas, das Ihnen jetzt in der Krise besonders hilft? gingen in 88 Fragebogen Antworten ein, die sich in folgende Gruppen zusammenfassen ließen (- Tab. 3).

Neben dem stützenden Erleben durch Kontakte und Gespräche in der Familie steht die Erfahrung eines sozialen Miteinanders im Vordergrund. Weiter nennen die Befragten neben „Wissen und Lebenserfahrung" häufig positive Lebenseinstellungen als hilfreich. Sie zeigen sich gelassen und optimistisch und geben an, dass die Kriegserfahrung ihnen helfe, das Erleben der Kontakteinschränkung zu relativieren: „Wir hungern und frieren ja nicht". Natur und Hobby sind weitere häufig genannte Kraftquellen.

\section{Verändern sich die Befunde beim zweiten Lockdown?}

In der zweiten Umfrage im Dezember 2020 ergibt sich im Wesentlichen eine Konstanz der Befunde. Die größten Unterschiede gab es bei der Frage 6 (Ist Ihnen die Kontakteinschränkung schwergefallen? - hier stieg der Mittelwert um 0,4 auf $m=3,89$ ) und bei der Frage 13 (Empfinden Sie die Coronakrise im Vergleich zur Kriegs- und Nachkriegszeit als harmlos? - hier sank der Mittelwert um 0,4 auf $m=2,57$ ).

Die Dauer der Krise macht den Zeitzeugen deutlich zu schaffen $(m=3,75)$. Einsamkeit scheint eher nicht das zentrale Problem zu sein $(m=2,33)$. Die meisten Zeitzeugen wollen sich impfen lassen $(m=4,0)$. Mittlere Zustimmung erfahren Fragen nach einer besonderen Belastung durch die bevorstehenden Weihnachtstage $(m=2,69)$ oder durch das Silvesterfeuerwerk $(m=2,33)$. 


\section{Erleben der Coronakrise}

vor dem Hintergrund der

Feuersturmerfahrung

Bei dem Item 11 (Erinnert die gegenwärtige Krise Sie an Ihre Erfahrungen im Hamburger Feuersturm?) antworteten $13 \mathrm{Be}-$ fragte auf der höchsten Stufe (5) mit; Ja. Von innen wurde bei 11 Befragten dieses Item in der zweiten Befragung erneut auf der höchsten Stufe beantwortet, in einem Fall ist es aber von 5 auf 4 gesunken, und in einem anderen Fall von 5 auf 2. Die Korrelationsberechnungen mit den anderen Items des Fragebogens (Spalte 3 und 7 der 0 Tab. 1) zeigen zu beiden Zeitpunkten bei 3 Variablen eine dem Betrag nach kleine, jedoch signifikante Korrelation mit den Antworten auf Frage 11 ( $p$-Wert $<0,05$ ): Schlafprobleme $(0,26)$ bzw. $(0,32)$, übertriebene Ängste $(0,28)$ bzw. $(0,40)$, Eindruck einer Nützlichkeit der Kriegserfahrungen für die persönliche Bewältigung der Coronakrise $(0,26)$ bzw. $(0,24)$.

Eine stark signifikante Korrelation $(0,58)$ bzw. $(0,64)$ ( $p$-Wert $<0,001)$ ergibt sich für die Frage nach dem Erinnertwerden an die Nachkriegszeit. Allerdings korrelieren hier meist die niedrigen Werte; die Befragten, die sich durch die Coronakrise nicht an den Hamburger Feuersturm erinnert fühlten, sahen sich auch nicht an die Nachkriegszeit erinnert. Eine weitere signifikante (negative) Korrelation mit dem Erleben, die Coronakrise sei im Vergleich zur Kriegsund Nachkriegszeit harmlos $(-0,3)$, zeigt sich in der zweiten Befragung nicht mehr $(-0,1)$.

\section{Bezüge zu den Inhalten der lebensgeschichtlichen Interviews}

Es stellte sich nun die Frage, welche konkreten Erfahrungen im Feuersturm die 13 Rücksender gemacht haben, die auf die Frage 11 (Erinnert die gegenwärtige Krise Sie an Ihre Erfahrungen im Hamburger Feuersturm?) auf der Endstufe der Skalierung $(j a=5)$ geantwortet haben. Dazu haben wir in unserer Forschungsgruppe die in den im Erinnerungswerk bereits vorliegenden transkribierten Interviews dieser Rücksender herangezogen und ad hoc zentrale Grundzüge der geschilderten Erfahrungen im Feuersturm und der Kriegs- und Nachkriegszeit auf einer Skala von 0 bis 3 gemeinsam eingeschätzt. Dabei zeigte sich eine massive Belastung dieser Zeitzeugen weit über die eigentliche Todesbedrohung im Feuersturm $(m=1,96)$ hinaus. Im Vordergrund stehen hier die Folgen der Ausbombung mit der damit verbundenen Entwurzelung $(m=2,38)$, neben weiteren Nöten und Entbehrungen in der Kriegszeit $(m=1,92)$ und der Nachkriegszeit $(m=1,92)$. Nur ein Zeitzeuge dieser Gruppe war vergleichsweise gering belastet.

\section{Diskussion}

Unsere explorative Querschnittsstudie gibt einen ersten Einblick in das Erleben der Coronakrise in der Generation der "Kriegskinder des Zweiten Weltkriegs". Der hohe Rücklauf der Fragebogen in der Gruppe der Hochbetagten $(81,7 \%$, $64 \%$ spricht für die subjektive Relevanz der Fragestellung bei den Befragten und ihre Bindung an das Erinnerungswerk. Insgesamt zeigen sich die Überlebenden des Hamburger Feuersturms am Anfang des ersten Coronalockdowns im Frühjahr 2020 wie auch im Dezember 2020 überwiegend gelassen und stabil. Gleichzeitig wird deutlich, dass die verhängten Kontaktbeschränkungen, besonders die Einschränkung des unmittelbaren auch körperlichen Kontakts in der Familie erheblich zu schaffen machen. Die Sorge um die eigene Gesundheit und die Angst vor Ansteckung ist weniger ausgeprägt. Als ein wesentliches Element gut durch die Krise zu kommen, wird eine positive eigene Einstellung gesehen. Möglicherweise werden hier Haltungen im Sinne einer "generationsbedingten Genügsamkeit" [6] aktiviert, die in der Kriegszeit ausgebildet wurden (z. B. Optimismus als Überlebensprinzip, Ruhe bewahren), aber auch Erfahrung und Wissen werden als wichtig erachtet. Freilich finden wir in den Fragebogen auch eine Untergruppe von ca. $13 \%$ der Antwortenden, die einen erinnernden Bezug zwischen der Feuersturm und Coronaerfahrungen angeben. Unsere Befunde sprechen insgesamt dafür, dass bei schwer betroffenen "Kriegskindern" des II. Weltkriegs die Kriegserfahrungen in inrem Selbsterleben als stabilisierendes inneres Referenzsystem zur Bewältigung und Relativierung der Coronakrise dienen können, dass aber auch schmerzhaftes und schwieriges "Wiedererinnern" vorkommt.

\section{Ausblick}

Es erscheint sinnvoll, sich gezielt dem zeitgeschichtlich geprägten Erleben in der Altersgruppe der Hochbetagten zuzuwenden. Dies erscheint umso notwendiger, als z.B. eine groß angelegte nationale Studie zum Erleben der Coronakrise die über 79-Jährigen nicht einbezieht [8]. Ihre kollektive Benennung als "Hochrisikogruppe" kann diskriminierend wirken und die „Krisenfestigkeit" der einzelnen Menschen überblenden [1].

\section{Fazit für die Praxis}

- Die befragten Überlebenden des Hamburger Feuersturms schildern sich und ihre persönliche Situation als überwiegend stabil.

- Vor allem die mit dem Lockdown verbundenen Kontaktbeschränkungen werden als belastend erlebt.

- Die Kriegserfahrung hilft zur Relativierung der gegenwärtigen Krise.

- Eine Untergruppe erlebt starke Bezüge zur Feuersturmerfahrung und zu anhaltenden Belastungen in der Kriegs- und Nachkriegszeit.

- Generell sollten die Erfahrungen im Zweiten Weltkrieg in der psychosozialen Betreuung der heute über 80 -Jährigen berücksichtigt werden.

\section{Korrespondenzadresse}

\section{PD Dr. Ulrich Lamparter}

Adolf-Ernst-Meyer-Institut für Psychotherapie Rothenbaumchaussee 71, 20148 Hamburg, Deutschland

ulamparter@t-online.de

Förderung. Die Untersuchung wurde gefördert mit Mitteln des Michael-Balint-Instituts Hamburg, des Adolf-Ernst-Meyer-Instituts für Psychotherapie und des Instituts für Psychosomatische Medizin und Psychotherapie des Universitätsklinikums HamburgEppendorf sowie des Instituts für Verhaltenstherapie Ausbildung (IVAH) Hamburg.

\section{Einhaltung ethischer Richtlinien}

Interessenkonflikt. U. Lamparter, H. Althoff, C. Holstein, S. Lucassen, U. Pilz und U. Rippel-Lau geben an, dass kein Interessenkonflikt besteht. 
Im Erinnerungswerk EHF-1943 liegen weitreichende Einverständniserklärungen zur wissenschaftlichen Auswertung und Publikation vor.

\section{Literatur}

1. Kricheldorff C (2020) Gesundheitsversorgung und Pflege für ältere Menschen in der Zukunft. Erkenntnisse aus der Corona-Pandemie.ZGerontol Geriat 53:742-748

2. Lamparter U, Holstein C (2015) Gebranntes Kind für immer? - Qualitative und quantitative Befunde zur Frage der Traumatisierung bei Zeitzeugen des "Hamburger Feuersturms" (1943). Psyche Z Psychoanal 69:161-187

3. Lamparter U, Holstein C, Thießen M, Wierling D, Wiegand-Grefe S, Möller B (2010) 65 Jahre später. Zeitzeugen des „Hamburger Feuersturms“ (1943) im lebensgeschichtlichen Interview. Forum Psychoanal 26(4):365-387

4. Lamparter U, Wiegand-Grefe S, Wierling D (Hrsg) (2013) Zeitzeugen des Hamburger Feuersturms 1943 und ihre Familien. Vandenhoeck \& Ruprecht, Göttingen

5. Lamparter U, Drost N, Nickel S (2015) „Hier und überall lechzten die Flammen und es brüllte die Luft" - eine systematisierte diagnostische Eindrucksbildung an Überlebenden des „Hamburger Feuersturms" (1943). Z Psychosom Psychother 61:173-190

6. Nikelski A, Trompetter E, Feldmann S, Whittacker E-S, Boekholt M, Chikradze N, Kracht F, Lücker P, Vollmar HC, Thyrian JR, Kreisel S (2021) „Das muss man so nehmen." Eine Studie zum subjektiven Erleben der Coronapandemie älterer hilfe-pflegebedürftiger Menschen in der Häuslichkeit. Z Gerontol Geriat. https://doi.org/10.1007/ s00391-021-01888-6

7. Overy R (2014) Der Bombenkrieg. Europa 1939-1945. Rowohlt, Berlin

8. Peters A, Rospleszcz S, Greiser H, Dallavalle $M$, Berger K (2020) COVID-19-Pandemie verändert die subjektive Gesundheit. Erste Ergebnisse der NAKO-Gesundheitsstudie. Dtsch Arztebl 117(50):861-869

9. Radebold H (Hrsg) (2004) Kindheiten im II. Weltkrieg und ihre Folgen. Psychosozial-Verlag, Gießen

10. Thiessen M (2007) Eingebrannt ins Gedächtnis. Hamburgs Gedenken an Luftkrieg und Kriegsende 1943 bis 2005. Dölling und Galitz, Hamburg

\section{Do the old fears return? An investigation into the experience of the corona crisis among survivors of the Hamburg Firestorm (1943)}

Background: The generation of war children of the Second World War is currently in old age experiencing the lock-down caused by the coronavirus crisis.

Objective: How are the restrictions of the lock-down experienced against the background of the war experience?

Methods: A total of 120 witnesses of the Hamburg Firestorm (1943) were asked about their experiences of the corona pandemic by means of a questionnaire in May 2020 and December 2020. Findings from telephone conversations with several witnesses, who regularly participate in a discussion group, have also been taken into consideration for this study.

Results: Of the interviewees contacted in May 2020 and December 2020, 98 (82\%) and 77 (64\%), respectively, sent back the questionnaire, 58 (45) female and 40 (32) male, the mean age was 86.5 years ( 87.1 years). According to the questionnaire most of them feel relatively stable and confident about their general situation in the pandemic and are mostly concerned with the contact restrictions rather than with their own health. The majority fear negative economic consequences for Germany. About 13\% fully agree that the current crisis reminds them of their experiences in the Hamburg Firestorm. As telephone conversations have shown the memories and experiences of the war and the post-war period in general, seem to act as the leading frame of reference for dealing with the current crisis.

Conclusion: The findings point to typical psychological processing patterns in a warburdened generation, when they now relate their experiences in the war to the experiences in the corona crisis.

\section{Keywords}

Corona crisis · Air war · Psychological consequences of the Second World War · Coping 